\title{
Phased Array Beamforming and Imaging in Composite Laminates Using Guided Waves
}

\author{
Zhenhua Tian ${ }^{1 *}$, Cara A.C. Leckey ${ }^{2}$, Lingyu Yu ${ }^{1}$ \\ ${ }^{1}$ Department of Mechanical Engineering, University of South Carolina, Columbia, SC \\ ${ }^{2}$ Nondestructive Evaluation Sciences Branch, NASA Langley Research Center, Hampton, VA
}

\begin{abstract}
This paper presents the phased array beamforming and imaging using guided waves in anisotropic composite laminates. A generic phased array beamforming formula is presented, based on the classic delay-and-sum principle. The generic formula considers direction-dependent guided wave properties induced by the anisotropic material properties of composites. Moreover, the array beamforming and imaging are performed in frequency domain where the guided wave dispersion effect has been considered. The presented phased array method is implemented with a non-contact scanning laser Doppler vibrometer (SLDV) to detect multiple defects at different locations in an anisotropic composite plate. The array is constructed of scan points in a small area rapidly scanned by the SLDV. Using the phased array method, multiple defects at different locations are successfully detected. Our study shows that the guided wave phased array method is a potential effective method for rapid inspection of large composite structures.
\end{abstract}

Keywords: guided waves; phased arrays; anisotropic composites; array imaging; multiple defects; rapid inspection

\section{INTRODUCTION}

Advanced composite materials will likely play an important role in NASA's development of next generation vehicles for long duration space missions in order to enable lightweight space structures. NASA is currently investigating large scale composites for human spaceflight, such as a composite crew module and a composite fuel tank for rockets [1]. Rapid inspection techniques for detecting and quantifying damage in large composites are critical for ensuring operability and safety of composite structures $[2,3]$. Though various damage inspection methods have been developed for metallic structures, reliable and efficient evaluations of large composite structures are not yet well established [3, 4].

For rapid damage inspection, an image of the structure being inspected often gives an efficient solution that quickly identifies and locates defects. Among various imaging methods, the guided wave phased array imaging is attractive since it uses sensors that are placed closed to each other in a compact format, steers the outputs of all sensors in a desired direction, and scans the entire structure like a radar [5], allowing for large area inspection with limited access. Intensive study has been conducted on the guided wave phased array beamforming and damage detection on metallic plate like structures [5-33]. Some researchers have started investigating the phased arrays for anisotropic composite materials [3439]. It has been found that the guided wave complexity caused by anisotropic and inhomogeneous properties in composite materials makes the traditional metal-based methods inappropriate and sometimes even misleading [40-42].

In this paper, we investigate guided wave beamforming in anisotropic laminated composite plates. Based on the classic delay-and-sum principle, a generic formula of phased array beamforming in anisotropic composite plates is developed, in which the direction dependent guided wave properties are considered. Moreover, the array beamforming and imaging are performed in frequency domain where the guided wave dispersion effect has been considered. The presented phased array method is implemented with a non-contact scanning laser Doppler vibrometer (SLDV). For the proof of concept, laboratory tests are performed using rectangular grid arrays made of scanning points of the non-contact SLDV for detecting multiple surface defects. The results show that multiple defects at various directions can be successfully detected using the non-contact SLDV array. The remainder of this paper is organized as follows: Section 2 presents the formulation of phased array beamforming in anisotropic composite plates; Section 3 presents the

\footnotetext{
* Corresponding author. Email: tianz@email.sc.edu
} 
implementation of phased arrays by using a non-contact SLDV for detecting multiple defects in a composite plate; Section 4 concludes the paper with novelties, discussions and planned future work.

\section{GUIDED WAVE BEAMFORMING IN COMPOSITE LAMINATES}

This section presents the guided wave beamforming in anisotropic composite laminates. The generic beamforming formulas have been derived based on the classic delay-and-sum principle. Compared to the array beamforming of circular wavefront guided waves in isotropic materials, the noncircular wavefront characteristic of guided waves in anisotropic materials is considered in the generic beamforming formulas.

\subsection{Guided waves in composite laminates}

When a guided wave with frequency $\omega$ and wavenumber $\mathbf{k}$ is generated from a source at the coordinate origin $O$ in a composite plate, the wave arriving at the location $\mathbf{x}$ that is far away from the source (Figure 1a) can be expressed as [4244],

$$
u(t, \mathbf{x})=A e^{j(\omega t-\mathbf{k} \cdot \mathbf{x})}
$$

where $A$ is the amplitude, assumed to be independent of wave frequency. With the geometric relation illustrated in Figure 1a, we have,

$$
\mathbf{k} \cdot \mathbf{x}=|\mathbf{k}||\mathbf{x}| \cos \beta=k(\gamma)|\mathbf{x}| \cos \beta
$$

with $\beta$ being the angle between the wave propagation and wavenumber $\mathbf{k}$. Hence,

$$
u(t, \mathbf{x})=A e^{j[\omega t-k(\gamma)|\mathbf{x}| \cos \beta]}
$$

Using Eq.(1), for a source located at location $\mathbf{p}_{m}$, the wave resulted at the location $\mathbf{x}$ is,

$$
u_{m}(t, \mathbf{x})=A e^{j\left[\omega t-\mathbf{k} \cdot\left(\mathbf{x}-\mathbf{p}_{m}\right)\right]}
$$

In anisotropic composite laminates, guided wave parameters such as wavenumbers, phase velocities and group velocities are direction dependent, due to the direction dependent physical properties of composite materials [40-42]. Figure 1b plots the wavenumber curve $k(\gamma)$. As illustrated in Figure $1 \mathrm{~b}$, the wavenumber vector $\mathbf{k}$ is perpendicular to the wave front and the group velocity vector $\mathbf{c}_{\mathrm{g}}$ is orthogonal to the wavenumber curve $k(\gamma)$ [40-42]. The angle $\gamma$ of the wavenumber vector $\mathbf{k}$ is referred to as wavenumber angle. The angle $\theta$ of the group velocity vector $\mathbf{c}_{\mathrm{g}}$ is referred to as group velocity angle (or energy propagation angle). The angle $\beta$ between wavenumber angle $\gamma$ and energy propagation angle $\theta$ is referred to as skew angle, with the relation $\beta=\gamma-\theta$. It can be seen when $\mathbf{c}_{\mathrm{g}}$ is not parallel to $\mathbf{k}$, the skew angle $\beta$ is not zero and hence the wave energy propagation direction is not perpendicular to the wave front.

(a)

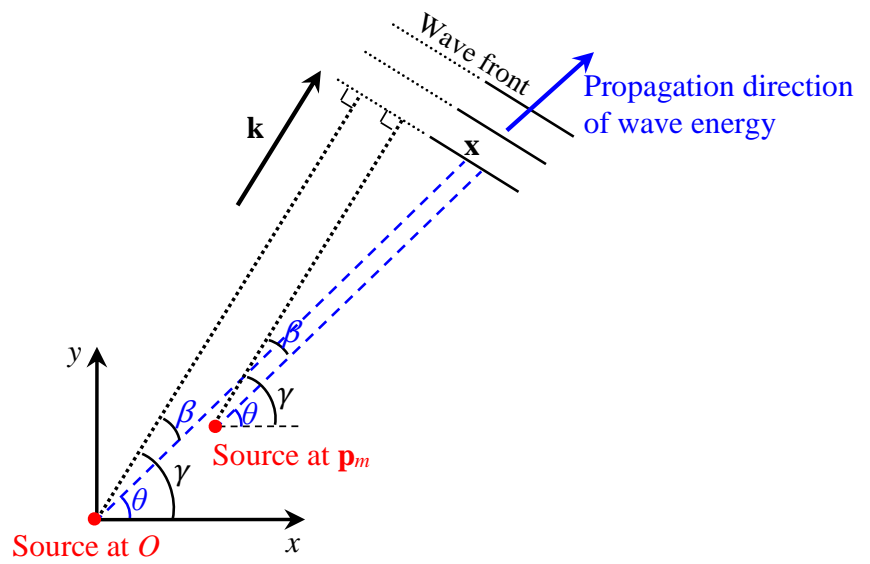

(b)

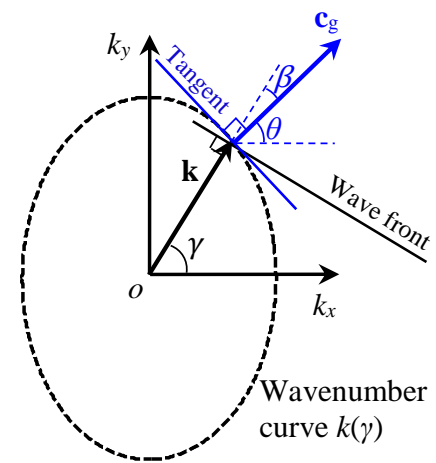

Figure 1 Schematics of geometric relations of guided waves in composite laminates: (a) the geometric relation of GW (in the far field) generated by sources at different locations; (b) wavenumber curve. The geometric relation between the wavenumber vector $\mathbf{k}$ and the group velocity vector $\mathbf{c}_{\mathrm{g}}$ is given in figure $\mathrm{b}$. 


\subsection{Delay-and-sum beamforming}

Consider an array with $M$ identical elements located at $\left\{\mathbf{p}_{m}\right\}(m=0,1,2, \ldots M-1)$ which are geometrically close to each other. The phase center is defined as the origin $O$ of the Cartesian coordinate system, i.e., $1 / M \sum_{m=0}^{M-1} \mathbf{p}_{m}=\mathbf{0}$. Each element serves as a wave source. When all elements generate waves with frequency $\omega$ and wavenumber vector $\mathbf{k}$ simultaneously, using Eq. (4) the total output (synthesized wave) of the array at location $\mathbf{x}$ can be derived as,

$$
z(t, \mathbf{x})=\sum_{m=0}^{M-1} A e^{j\left[\omega t-\mathbf{k} \cdot\left(\mathbf{x}-\mathbf{p}_{m}\right)\right]}=u(t, \mathbf{x}) \sum_{m=0}^{M-1} e^{j \mathbf{k} \cdot \mathbf{p}_{m}}
$$

It is seen from Eq. (5) that the synthesized wave $z(t, \mathbf{x})$ is an amplification of the wave $u(t, \mathbf{x})$ emitted from the Origin. The amplification is controlled by the exponential component $\sum_{m=0}^{M-1} e^{j \mathbf{k} \cdot \mathbf{p}_{m}}$ in Eq. (5). Therefore, by adjusting the component $\sum_{m=0}^{M-1} e^{j \mathbf{k} \cdot \mathbf{p}_{m}}$, we can control the amplification. One way to adjust the component $\sum_{m=0}^{M-1} e^{j \mathbf{k} \cdot \mathbf{p}_{m}}$ is applying phase delays to all exponents. For example, to maximize the amplification $\sum_{m=0}^{M-1} e^{j \mathbf{k} \cdot \mathbf{p}_{m}}$ in a specific direction $\theta_{S}$, we can apply phase delays $\Delta_{m}\left(\theta_{S}\right)$ to each exponent to make each exponent zero,

$$
\sum_{m=0}^{M-1} e^{j\left[\mathbf{k} \cdot \mathbf{p}_{m}-\Delta_{m}\left(\theta_{S}\right)\right]}=\sum_{m=0}^{M-1} e^{j 0}=M, \text { where } \Delta_{m}\left(\theta_{S}\right)=\mathbf{k} \cdot \mathbf{p}_{m}
$$

Using phase delays $\Delta_{m}\left(\theta_{S}\right)$, the amplification is maximized at the direction $\theta_{S}$, i.e., generating a directional "beam". $\theta_{S}$ is also known as steering angle. Eq. (6) shows that phase delays $\Delta_{m}\left(\theta_{S}\right)$ depend on the $m^{\text {th }}$ element's position vector $\mathbf{p}_{m}$ and wavenumber vector $\mathbf{k}$.

In anisotropic composites, the wavenumber vector $\mathbf{k}$ depends on wave frequency $\omega$ and wavenumber angle $\gamma_{S}$. In addition, the geometry relation between wavenumber angle $\gamma_{S}$ and steering angle $\theta_{S}$ is $\gamma_{S}=\theta_{S}+\beta_{S}$, where $\beta_{S}$ is referred to as skew angle. Hence, phase delays $\Delta_{m}\left(\theta_{S}\right)$ in Eq.(6) can be further expressed as,

$$
\Delta_{m}\left(\theta_{S}\right)=\mathbf{k}\left(\omega, \theta_{S}+\beta_{S}\right) \cdot \mathbf{p}_{m}
$$

It can be seen from Eq.(7) that the phase delay includes a frequency dependent term, $\mathbf{k}\left(\omega, \theta_{S}+\beta_{S}\right)$. Hence, it is frequency dependent and has the advantage of compensating dispersion effects (a.k.a. frequency dependent wave properties) by using frequency-related components during the delaying [14], [45]. This is otherwise not readily achievable in the commonly used time delay methods [5, 11,34].

In addition to phase delays, weighting factors $w_{m}$ can also be applied to delayed waves to further control the quality of beamforming $[43,46]$. In summary, with phase delays and weighting factors, the beamforming is represented as,

$$
z(t, \mathbf{x})=u(t, \mathbf{x}) \sum_{m=0}^{M-1} w_{m} e^{j\left[\mathbf{k} \cdot \mathbf{p}_{m}-\Delta_{m}\left(\theta_{s}\right)\right]}=u(t, \mathbf{x}) \sum_{m=0}^{M-1} w_{m} e^{j\left[\mathbf{k}-\mathbf{k}\left(\omega, \theta_{S}+\beta_{S}\right)\right] \cdot \mathbf{p}_{m}}
$$

\subsection{Array beamforming in a CFRP composite plate}

To give a direct view and better understanding of the array beamforming in anisotropic composites, wavefields are simulated by using equations given in subsection 2 . The simulation specimen is an 8-ply $\left[\mathrm{O}_{2} / 90_{2}\right]_{\mathrm{s}}$ CFRP composite plate. The material properties for a single ply are given in Table 1 . The $\mathrm{A}_{0}$ mode at $90 \mathrm{kHz}$ is chosen for the simulation (its wavenumber curve is plotted in Figure 2).

Figure 3a plots the wavefield calculated using Eq. (1), in which the amplitude $A(\omega)$ is assumed to be unity. This wavefield is for the $90 \mathrm{kHz} \mathrm{A}$ mode generated by a point source at the coordinate origin in the $\left[0_{2} / 90_{2}\right]_{\mathrm{s}} \mathrm{CFRP}$ plate. As shown in the wavefield in Figure 3a, waves propagate to all directions from the actuator at the coordinate origin. Figure $3 \mathrm{~b}$ plots the synthesized wavefield calculated using Eq. (8). This wavefield represents guided waves generated from a rectangular grid array $(16 \times 16$ elements with the array spacing $2 \mathrm{~mm})$, when the wave energy steering angle $\theta_{S}$ is $45^{\circ}$. Compared to the wavefield in Figure 3a, the synthesized wavefield in Figure $3 \mathrm{~b}$ generated from the array shows directional guided waves in the direction $\theta=45^{\circ}$. 
Table 1 Material properties

\begin{tabular}{lllllllllll}
\hline$\rho\left(\mathrm{kg} / \mathrm{m}^{3}\right)$ & $\mathrm{E}_{1}(\mathrm{GPa})$ & $\mathrm{E}_{2}(\mathrm{GPa})$ & $\mathrm{E}_{3}(\mathrm{GPa})$ & $\mathrm{G}_{12}(\mathrm{GPa})$ & $\mathrm{G}_{13}(\mathrm{GPa})$ & $\mathrm{G}_{23}(\mathrm{GPa})$ & $v_{12}$ & $v_{13}$ & $v_{23}$ & Thickness $(\mathrm{mm})$ \\
\hline 1570 & 171.4 & 9.08 & 9.08 & 5.29 & 5.29 & 2.80 & 0.32 & 0.32 & 0.34 & 0.1068
\end{tabular}

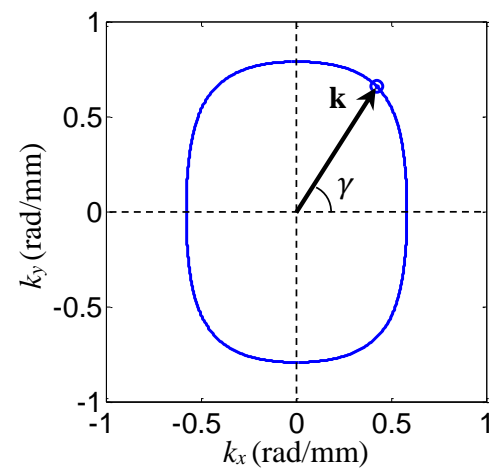

Figure 2 The wavenumber curve of $\mathrm{A}_{0}$ mode at $90 \mathrm{kHz}$ in the composite laminate with $\left[0_{2} / 90_{2}\right]_{\mathrm{s}}$ layup
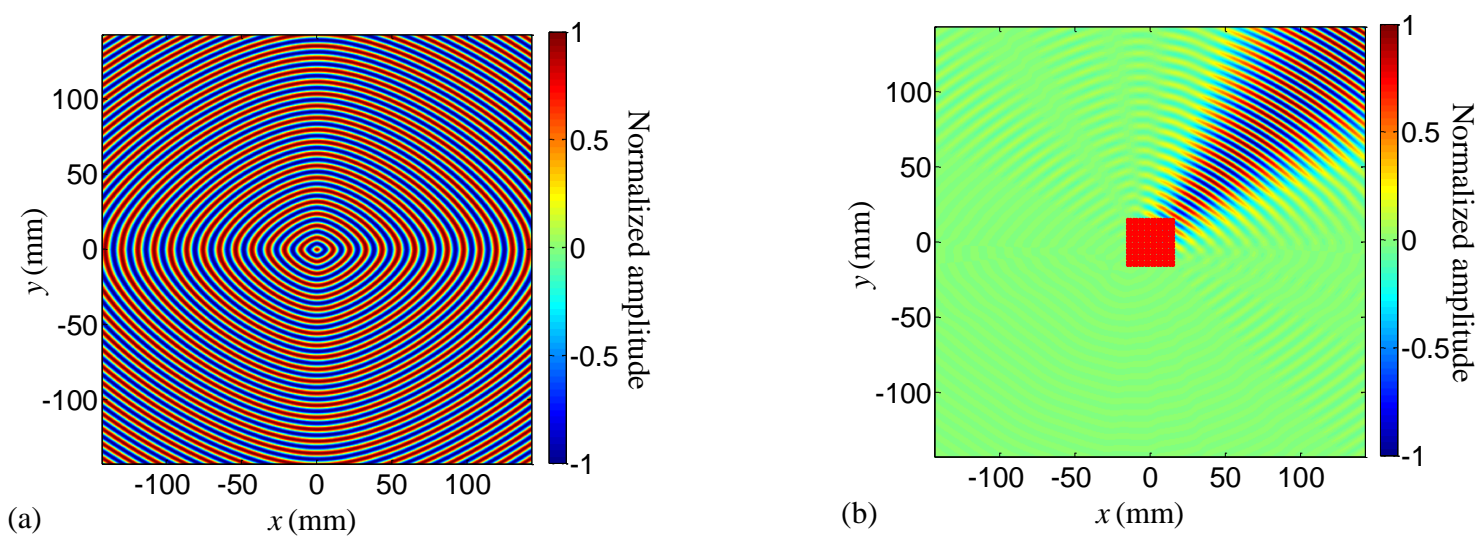

Figure 3 Simulated wavefields of the $90 \mathrm{kHz} \mathrm{A}_{0}$ mode in a composite plate with $\left[0_{2} / 90_{2}\right] \mathrm{s}$ layup: (a) generated by a single point source at the origin, (b) generated by rectangular grid array $\left(16 \times 16\right.$ elements) when the wave steering angle $\theta_{S}$ is $45^{\circ}$.

\section{DETECTION OF MULTI-DEFECTS IN A CFRP COMPOSITE PLATE}

In this section, guided wave beamforming and damage detection are implemented for a laminated composite plate using rectangular grid arrays. A hybrid measurement PZT-SLDV system consisting of a surface bonded PZT wafer and a non-contact scanning laser Doppler vibrometer (SLDV) is used for guided wave actuation and sensing [47]. Phased arrays are constructed by selected scanning points of the SLDV. Four quartz rods are surface bonded on a composite plate at various angular positions as defects to be detected by the phased array method.

\subsection{Experimental setup}

Figure 4 plots a schematic of experimental setup for the PZT-SLDV phased array sensing. The test specimen is an 8-ply $\left[0_{2} / 90_{2}\right]_{s}$ layup composite laminate with dimensions of $380 \times 380 \times 0.85$ (unit: $\mathrm{mm}$ ). The material properties of a single ply are listed in Table 1 . The plate center is set as the coordinate origin. Four defects $\left(\mathrm{D}_{1}, \mathrm{D}_{2}, \mathrm{D}_{3}\right.$ and $\left.\mathrm{D}_{4}\right)$ are simulated by using four quartz rods with the same size bonded on the plate surface with the same distance $100 \mathrm{~mm}$ to the 
plate center while different angles $0^{\circ}, 45^{\circ}, 90^{\circ}$ and $135^{\circ}$. A PZT wafer (APC 851: $7 \mathrm{~mm}$ diameter, $0.2 \mathrm{~mm}$ thickness) is bonded on the plate center to generate guided waves. A 3-cycle toneburst with the frequency $90 \mathrm{kHz}$ is generated from the function generator (model: Agilent 33522B), amplified to $30 \mathrm{~V}$ by a voltage amplifier (model: Krohn-Hite 1506), and applied to the PZT wafer for excitation. An SLDV (model: Polytec PSV-400-M2) is used to acquire velocity components of guided waves at selected scanning points, based on Doppler Effect. The laser beam is set perpendicular to the plate such that the out-of-plane velocity of guided waves is measured.

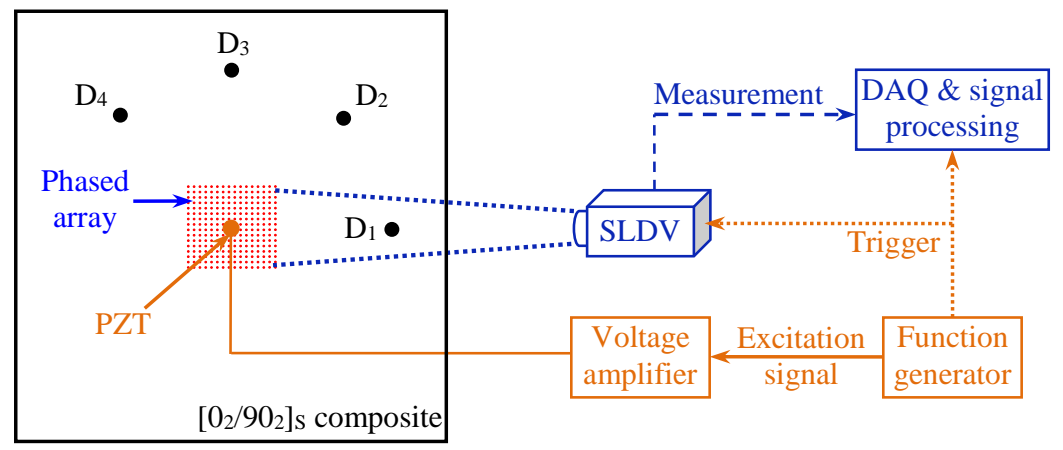

Figure 4. The schematic of experimental setup for multi-defects detection in a $\left[0_{2} / 90_{2}\right]_{\mathrm{S}}$ CFRP composite laminate.

\subsection{Array imaging algorithms}

The phased array is constructed of SLDV scanning points at selected locations $\left\{\mathbf{p}_{m}\right\}(m=0,1,2, \ldots M-1)$, whose phase center satisfies $1 / M \sum_{m=0}^{M-1} \mathbf{p}_{m}=\mathbf{0}$. At the $\mathrm{m}^{\text {th }}$ array point $\left(\mathbf{p}_{m}\right)$, the acquired signal by the SLDV is denoted as $v_{m}(t)$. The signal's frequency spectrum is obtained using the Fourier transform, as:

$$
V_{m}(\omega)=\mathrm{F}\left[v_{m}(t)\right]=\int_{-\infty}^{\infty} v_{m}(t) e^{-j \omega t} d t
$$

Using the frequency spectrum $V_{m}(\omega)$, the synthesized frequency-space representation $Z(\omega, \mathbf{x})$ of the array [14] is:

$$
Z(\omega, \mathbf{x})=\sum_{m=0}^{M-1} w_{m} V_{m}(\omega) e^{j\left[-\varphi(\omega, \mathbf{x})-\Delta_{m}(\omega, \mathbf{x})\right]}
$$

where,

$$
\Delta_{m}(\omega, \mathbf{x})=\mathbf{k}(\omega, \gamma) \cdot \mathbf{p}_{m}, \text { and } \varphi(\omega, \mathbf{x})=-2 \mathbf{k}(\omega, \gamma) \cdot \mathbf{x}
$$

$\Delta_{m}(\omega, \mathbf{x})$ is the phase delay applied to the $\mathrm{m}^{\text {th }}$ array point for beamsteering, and $\varphi(\omega, \mathbf{x})$ represents the spatial phase shift. As guided waves travel from the PZT to the damage and then back to the array, they undergo a spatial phase shift $\varphi(\omega$, $\mathbf{x})$. Thus, $-\varphi(\omega, \mathbf{x})$ is applied in Eq. (10) in order to compensate for the spatial phase shift. $\mathbf{k}(\omega, \gamma)$ is the wavenumber vector at the frequency $\omega$ and wavenumber angle $\gamma$, and is obtained from wavenumber dispersion curves. $\gamma$ is the wavenumber angle determined from the geometry relation as $\gamma=\theta+\beta$, where $\theta$ is the energy propagation angle and equals the angle of vector $\mathbf{x}$, i.e., $\theta=\angle \mathbf{x}$, and $\beta$ is the skew angle corresponding to the energy propagation angle $\theta$. Weighting factors $w_{m}$ can be used to further control the quality of the beamforming such as the beam shape [43, 46]. In this study, weighting factors are set equal to one.

Using the inverse Fourier transform, the frequency-space representation $Z(\omega, \mathbf{x})$ can be transformed back to the time-space domain, as:

$$
z(t, \mathbf{x})=\mathrm{F}^{-1}[Z(\omega, \mathbf{x})]=\frac{1}{2 \pi} \int_{-\infty}^{\infty} Z(\omega, \mathbf{x}) e^{j \omega t} d \omega
$$


where $z(t, \mathbf{x})$ is a synthesized time-space wavefield that represents the array beamforming in the time-space domain. An inspection image of the plate to detect/locate any existing damage is then acquired by building an intensity image with the pixel value at the location $\mathbf{x}$ defined as:

$$
I(\mathbf{x})=|z(t=0, \mathbf{x})|^{2}
$$

\subsection{Array imaging results}

In this test, a rectangular phased array is used for damage detection. The array is constructed of $31 \times 31$ SLDV scan points with the spacing $2 \mathrm{~mm}$ and the array center at the origin. Using the phased array method presented in subsection 3.2, an intensity image can be generated with the pixel definition given in Eq. (13). Figure 5a plots the imaging result when the sizes of four defects are $4 \mathrm{~mm}$ (defects are simulated by four quartz rods with the same diameter of $4 \mathrm{~mm}$ and length of $10 \mathrm{~mm}$ ). The defects show up as highlighted spots in the image providing a useful means to detect as well as to locate their positions. Hence the four defects are located by looking for the local maximum pixel value (normalized amplitude): $\mathrm{D}_{1}$ at $(99.0,0.8) \mathrm{mm}$ with errors of $(1.0,0.8) \mathrm{mm}, \mathrm{D}_{2}$ at $(74.4,69.3) \mathrm{mm}$ with errors of $(3.7,1.4) \mathrm{mm}, \mathrm{D}_{3}$ at $(0.2,101.0) \mathrm{mm}$ with errors of $(0.2,1.0) \mathrm{mm}$, and $\mathrm{D}_{4}$ at $(-71.9,69.9) \mathrm{mm}$ with errors of $(1.2,0.8) \mathrm{mm}$.

Besides the case where the all four defect are size $4 \mathrm{~mm}$, other cases with defect sizes increased to 6,8 and $10 \mathrm{~mm}$ are also investigated. The array imaging results are plotted in Figure 5b, c and d, which show that the four defects are successfully detected. For comparison purpose, the image amplitudes (local maximum normalized amplitude) at the four detected areas are extracted and plotted with respect to the damage size in Figure 6, showing increasing amplitude trends with the increase of damage size. Moreover, Figure 6 shows how the amplitude varies with the inspection angle. The plot for $0^{\circ}$ has the highest amplitude, while the plot for $135^{\circ}$ (close to the plot for $45^{\circ}$ ) gives the smallest amplitude. The damage at $0^{\circ}$ has the highest amplitude due to the fact that the guided waves have the strongest propagation along this direction in the subject composite plate. In contrast, the amplitudes in $45^{\circ}$ and $135^{\circ}$ are very weak due to the fact that guided waves in these two directions are weak.
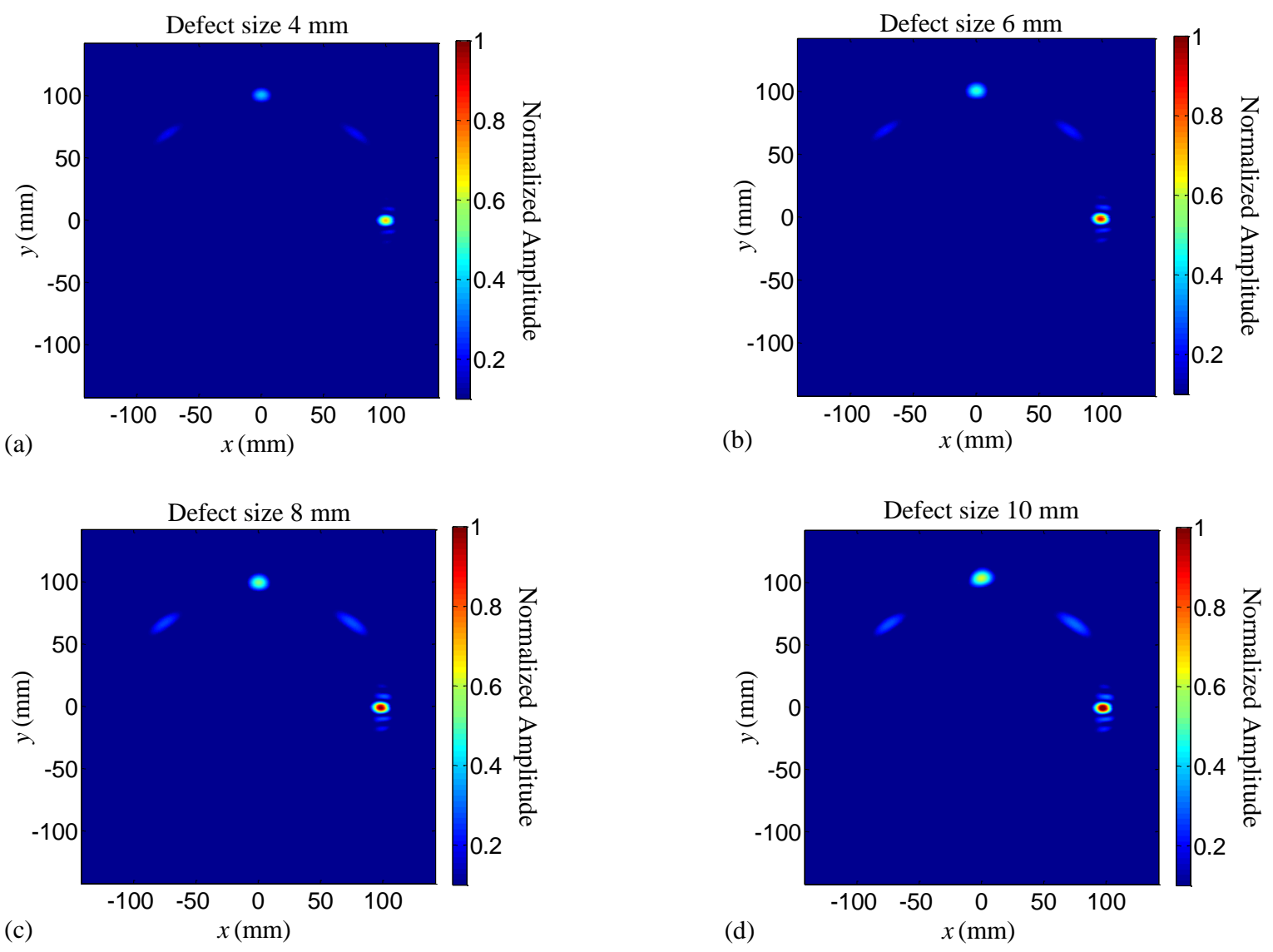
Figure 5. Array imaging results of the four defects. Sizes of defects are (a) $4 \mathrm{~mm}$, (b) $6 \mathrm{~mm}$, (c) $8 \mathrm{~mm}$ and (d) $10 \mathrm{~mm}$.

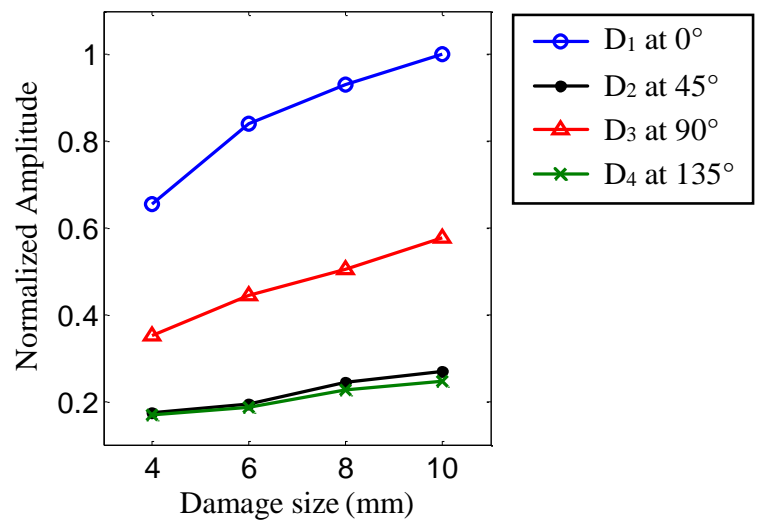

Figure 6. Image amplitude versus damage size. The four lines represent amplitudes at different inspection angles.

\section{CONCLUSIONS}

In this paper, we have presented a generic guided wave phased array beamforming method for rapid damage inspection in anisotropic composite laminates. The generic beamforming formula uniquely considers the directiondependent guided wave properties and the energy skew effect; thus it allows for phased array beamforming in anisotropic composites without requiring any quasi-isotropic assumption. The phased array beamforming is performed in the frequency domain which offers the advantage of compensating the guided wave dispersion effect through the use of frequency-dependent wavenumber.

The presented phased array method is implemented with a non-contact SLDV system which allows for the flexibility of using selected points from the SLDV scan to construct phase arrays of various configurations. The high spatial resolution feature of the SLDV system also significantly reduces array element spacing that gives the potential of using guided waves with smaller wavelengths to detect smaller defects in composite structures. We have shown that the present method can detect multiple defects at various locations in an anisotropic CFRP composite plate.

Despite the success of phased array beamforming and multiple defect detection in a composite plate presented here,

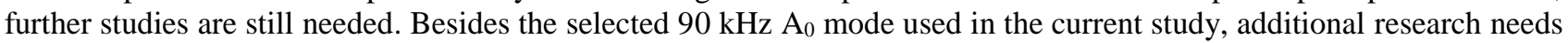
to be performed with guided waves at other frequencies and/or different wave modes in highly anisotropic composite plates. The effectiveness of the present method should be also evaluated with realistic defects such as impact induced delamination in composite plates, and studies of the smallest detectable defect size should be performed. The potential of weighting factors to improve the beamforming quality could also be investigated.

\section{ACKNOWLEDGEMENT}

The authors would like to thank (1) the non-reimbursement space act umbrella agreement SAA1-18124 between South Carolina Research Foundation (SCRF) and the National Aeronautics and Space Administration (NASA) Langley Research Center, and (2) SC NASA EPSCoR Research Grant Program 521192-USCYu.

\section{REFERENCES}

[1] Composite Crew Module (Ccm) Pressure Vessel Pathfinder Development. http://www.nasa.gov/, 2010.

[2] Staszewski, W. J., Mahzan, S., and Traynor, R., "Health Monitoring of Aerospace Composite Structures Active and Passive Approach," Compos Sci Technol, 69, 1678-1685 (2009).

[3] Giurgiutiu, V., and Soutis, C., "Enhanced Composites Integrity through Structural Health Monitoring," Appl Compos Mater, 19, 813-829 (2012). 
[4] Rose, J. L., "Successes and Challenges in Ultrasonic Guided Waves for Ndt and Shm," National Seminar \& Exhibition on Non-Destructive Evaluation, Tiruchirappalli, India. 1-11.

[5] Yu, L., and Giurgiutiu, V., "In Situ 2-D Piezoelectric Wafer Active Sensors Arrays for Guided Wave Damage Detection," Ultrasonics, 48, 117-134 (2008).

[6] Ambrozinski, L., Stepinski, T., and Uhl, T., "Efficient Tool for Designing 2d Phased Arrays in Lamb Waves Imaging of Isotropic Structures," J Intel Mat Syst Stronline (2014).

[7] Engholm, M., and Stepinski, T., "Direction of Arrival Estimation of Lamb Waves Using Circular Arrays," Struct Health Monit, 10, 467-480 (2011).

[8] de Marchi, L., Moll, J., and Marzani, A., "A Sparsity Promoting Algorithm for Time of Flight Estimation in Guided Waves-Based Shm," EWSHM-7th European Workshop on Structural Health Monitoring, Nantes, France. 583-590.

[9] Engholm, M., Stepinski, T., and Olofsson, T., "Imaging and Suppression of Lamb Modes Using Adaptive Beamforming," Smart Materials and Structures, 20, 085024 (2011).

[10] Kwon, H. S., Lee, S. S., and Kim, J. Y., "Beam Pattern Improvement by Compensating Array Nonuniformities in a Guided Wave Phased Array," Smart Materials and Structures, 22, 085002 (2013).

[11] Giurgiutiu, V., and Bao, J., "Embedded-Ultrasonics Structural Radar for in Situ Structural Health Monitoring of Thin-Wall Structures," Struct Health Monit, 3, 121-140 (2004).

[12] Deutsch, W. A. K., Cheng, A., and Achenbach, J. D., "Self-Focusing of Rayleigh Waves and Lamb Waves with a Linear Phased Array," Res Nondestruct Eval, 9, 81-95 (1997).

[13] Yoo, B., Purekar, A. S., Zhang, Y., and Pines, D. J., "Piezoelectric-Paint-Based Two-Dimensional Phased Sensor Arrays for Structural Health Monitoring of Thin Panels," Smart Mater Struct, 19, 075017 (2010).

[14] Wilcox, P. D., "Omni-Directional Guided Wave Transducer Arrays for the Rapid Inspection of Large Areas of Plate Structures," Ieee T Ultrason Ferr, 50, 699-709 (2003).

[15] Fromme, P., Wilcox, P. D., Lowe, M. J. S., and Canvley, P., "On the Development and Testing of a Guided Ultrasonic Wave Array for Structural Integrity Monitoring," Ieee T Ultrason Ferr, 53, 777-785 (2006).

[16] Giridhara, G., Rathod, V. T., Naik, S., Roy Mahapatra, D., and Gopalakrishnan, S., "Rapid Localization of Damage Using a Circular Sensor Array and Lamb Wave Based Triangulation," Mech Syst Signal Pr, 24, 29292946 (2010).

[17] Jarmer, G. J. S., Flynn, E. B., and Todd, M. D., "Multi-Wave-Mode, Multi-Frequency Detectors for Guided Wave Interrogation of Plate Structures," Struct Health Monit, 13, 120-130 (2014).

[18] Kannajosyula, H., Lissenden, C. J., and Rose, J. L., "Analysis of Annular Phased Array Transducers for Ultrasonic Guided Wave Mode Control," Smart Materials and Structures, 22, 085019 (2013).

[19] Kim, D., and Philen, M., "Guided Wave Beamsteering Using Mfc Phased Arrays for Structural Health Monitoring: Analysis and Experiment," J Intel Mat Syst Str, 21, 1011-1024 (2010).

[20] Malinowski, P., Wandowski, T., Trendafilova, I., and Ostachowicz, W., "A Phased Array-Based Method for Damage Detection and Localization in Thin Plates," Struct Health Monit, 8, 5-15 (2009).

[21] Ostachowicz, W., Kudela, P., Malinowski, P., and Wandowski, T., "Damage Localisation in Plate-Like Structures Based on Pzt Sensors," Mech Syst Signal Pr, 23, 1805-1829 (2009).

[22] Prado, V. T., Higuti, R. T., Kitano, C., Martinez-Graullera, O., and Adamowski, J. C., "Lamb Mode Diversity Imaging for Non-Destructive Testing of Plate-Likestructures," Ndt\&E Int, 59, 86-95 (2013).

[23] Purekar, A. S., Pines, D. J., Sundararaman, S., and Adams, D. E., "Directional Piezoelectric Phased Array Filters for Detecting Damage in Isotropic Plates," Smart Mater Struct, 13, 838-850 (2004).

[24] Rajagopalan, J., Balasubramaniam, K., and Krishnamurthy, C. V., "A Single Transmitter Multi-Receiver (Stmr) Pzt Array for Guided Ultrasonic Wave Based Structural Health Monitoring of Large Isotropic Plate Structures," Smart Mater Struct, 15, 1190-1196 (2006).

[25] Romanoni, M., Gonella, S., Apetre, N., and Ruzzene, M., "Two-Dimensional Periodic Actuators for FrequencyBased Beam Steering," Smart Mater Struct, 18, 125023 (2009).

[26] Senesi, M., Xu, B., and Ruzzene, M., "Experimental Characterization of Periodic Frequency-Steerable Arrays for Structural Health Monitoring," Smart Mater Struct, 19, 055026 (2010).

[27] Seung, H. M., Kim, H. W., and Kim, Y. Y., "Development of an Omni-Directional Shear-Horizontal Wave Magnetostrictive Patch Transducer for Plates," Ultrasonics, 53, 1304-1308 (2013).

[28] Sundararaman, S., Adams, D. E., and Rigas, E. J., "Biologically Inspired Structural Diagnostics through Beamforming with Phased Transducer Arrays," Int J Eng Sci, 43, $756-778$ (2005). 
[29] Swift, C. I., Pierce, S. G., and Culshaw, B., "Generation of a Steerable Ultrasonic Beam Using a Phased Array of Low Power Semiconductor Laser Sources and Fiber Optic Delivery," Smart Mater Struct, 16, 728-732 (2007).

[30] Velichko, A., and Wilcox, P. D., "Guided Wave Arrays for High Resolution Inspection," J Acoust Soc Am, 123, 186-196 (2008).

[31] Wilcox, P. D., Lowe, M., and Cawley, P., "Omnidirectional Guided Wave Inspection of Large Metallic Plate Structures Using an Emat Array," Ieee T Ultrason Ferr, 52, 653-665 (2005).

[32] Higuti, R. T., Martinez-Graullera, O., Martin, C. J., Octavio, A., Elvira, L., and de Espinosa, F. M., "Damage Characterization Using Guided-Wave Linear Arrays and Image Compounding Techniques," Ieee T Ultrason Ferr, 57, 1985-1995 (2010).

[33] Koduru, J. P., Momeni, S., and Rose, J. L., "Phased Annular Array Transducers for Omnidirectional Guided Wave Mode Control in Isotropic Plate Like Structures," Smart Materials and Structures, 22, 8 (2013).

[34] Yan, F., and Rose, J. L., "Guided Wave Phased Array Beam Steering in Composite Plates," P Soc Photo-Opt Ins, 6532, G5320-G5320 (2007).

[35] Rajagopalan, J., Multila, K. H. M. O. A., Balasubramaniam, K., and Krishnamurthy, C. V., "A Phase Reconstruction Algorithm for Lamb Wave Based Structural Health Monitoring of Anisotropic Multilayered Composite Plates," J Acoust Soc Am, 119, 872-878 (2006).

[36] Vishnuvardhan, J., Muralidharan, A., Krishnamurthy, C. V., and Balasubramaniam, K., "Structural Health Monitoring of Anisotropic Plates Using Ultrasonic Guided Wave Stmr Array Patches," Ndt\&E Int, 42, 193-198 (2009).

[37] Leleux, A., Micheau, P., and Castaings, M., "Long Range Detection of Defects in Composite Plates Using Lamb Waves Generated and Detected by Ultrasonic Phased Array Probes," J Nondestruct Eval, 32, 200-214 (2013).

[38] Purekar, A. S., and Pines, D. J., "Damage Detection in Thin Composite Laminates Using Piezoelectric Phased Sensor Arrays and Guided Lamb Wave Interrogation," J Intel Mat Syst Str, 21, 995-1010 (2010).

[39] Osterc, P., Kim, D., and Yoo, B., "Phased Array Beamsteering in Composite Laminates for Guided Wave Structural Health Monitoring" ASME, Snowbird, Utah, USA(2013).

[40] Wang, L., and Yuan, F. G., "Group Velocity and Characteristic Wave Curves of Lamb Waves in Composites: Modeling and Experiments," Compos Sci Technol, 67, 1370-1384 (2007).

[41] Glushkov, E., Glushkova, N., and Eremin, A., "Group Velocity of Cylindrical Guided Waves in Anisotropic Laminate Composites," Journal of Acoustic Society of America, 135, 148-154 (2014).

[42] Nayfeh, A. H., "Wave Propagation in Layered Anisotropic Media" Elsevier, Amsterdam, The Netherlands(1995).

[43] Giurgiutiu, V., "Structural Health Monitoring with Piezoelectric Wafer Active Sensors" Academic Press, Boston, MA(2008).

[44] Rose, J. L., "Ultrasonic Waves in Solid Media" Cambridge University Press, New York(1999).

[45] Wilcox, P. D., "A Rapid Signal Processing Technique to Remove the Effect of Dispersion from Guided Wave Signals," Ieee T Ultrason Ferr, 50, 419-427 (2003).

[46] Johnson, D. H., and Dudgeon, D. E., "Array Signal Processing: Concepts and Techniques" Prentice-Hall Inc., Upper Saddle River, New Jersey, USA(1993).

[47] Yu, L., and Tian, Z., "Lamb Wave Structural Health Monitoring Using a Hybrid Pzt-Laser Vibrometer Approach," Structural Health Monitoring, 12, 469-483 (2013). 